Bundesgesundheitsbl 2010 · 53:643-652 DOI 10.1007/s00103-010-1083-2

Online publiziert: 4. Juni 2010

(c) Springer-Verlag 2010

\author{
B.-M. Kurth · A. Schaffrath Rosario \\ Robert Koch-Institut, Berlin
}

\title{
Übergewicht und Adipositas bei Kindern und Jugendlichen in Deutschland
}

\section{Methodische Grundlagen \\ (Referenzsysteme, Erhebungsmethoden)}

Adipositas und Übergewicht bei Kindern und Jugendlichen stellen in Deutschland wie auch in anderen europäischen Ländern eine zahlenmäßig bedeutsame Gesundheitsstörung dar, der zunehmend Aufmerksamkeit gewidmet wird. Obwohl sich in den letzten Jahren recht unterschiedliche Prävalenzschätzungen zu Adipositas und Übergewicht bei Kindern und Jugendlichen in der Fachliteratur finden ließen, war man sich doch darüber einig, dass ihre Häufigkeit besorgniserregend zugenommen hat [1]. Seit der Publikation der Ergebnisse des Kinderund Jugendgesundheitssurveys (KiGGS) gibt es deutschlandweit repräsentative Prävalenzaussagen. Die bislang berichteten Prävalenzen [2] beziehen sich auf die Altersgruppe drei bis 17 Jahre: 15\% der in Deutschland lebenden Kinder und Jugendlichen in dieser Altersgruppe sind nach dem Referenzsystem von Kromeyer-Hauschild et al. [3] übergewichtig, davon leiden 6,3\% an Adipositas. Wie diese Zahlen zu bewerten und wie sie zustande gekommen sind, auf welcher Datenbasis andere Einschätzungen beruhen und welche regionalen, sozialen und anderweitigen Differenzierungen möglich sind, soll in dieser Arbeit transparent gemacht werden. Auf dieser Grundlage kann bewertet werden, welche Entwicklungstrends bei Übergewicht und Adipositas bei Kindern und Jugendlichen $\mathrm{zu}$ erkennen sind und wie belastbar internationale Vergleiche sind.
Die epidemiologische Einschätzung der Übergewichts- und Adipositasproblematik auf Bevölkerungsebene basiert auf der Messung von Körpergröße und Körpergewicht. Damit unterscheidet sie sich von einer individuellen ärztlichen Diagnose, die neben Größe und Gewicht auch noch andere anthropometrische Maße, wie beispielsweise Hüft- und Taillenumfang und die Hautfaltendicke, berücksichtigt, aber auch Körperbau, Konstitution und Gesundheitszustand des jeweiligen Kindes in die Bewertung einbezieht. Nachfolgend geht es ausschließlich um die Epidemiologie, das heißt um die Verbreitung von Übergewicht und Adipositas in der Gruppe der Kinder und Jugendlichen. Die epidemiologischen Studien hierzu verwenden alle den Body-Mass-Index (BMI) zur Definition von Übergewicht und Adipositas.

\section{Der Body-Mass-Index \\ als Instrument zur \\ epidemiologischen Bewertung}

Der BMI, definiert als der Quotient aus dem in $\mathrm{kg}$ gemessenen Körpergewicht und dem Quadrat der in m gemessenen Körpergröße, hat sich in der Epidemiologie als Grundlage zur Definition von Übergewicht und Adipositas Erwachsener bewährt und wird auch für Kinder empfohlen [4, 5]. Auch wenn Messungen von Hautfaltendicken, des Taillenumfangs oder des daraus abgeleiteten Taille-
Größe-Quotients ebenfalls in der Literatur als Maße zur Definition von Übergewicht und Adipositas herangezogen werden, konnte in Studien keines dieser Maße als dem BMI eindeutig überlegen identifiziert werden $[6,7,8,9,10]$. Da zu den anderen Maßen von Kindern und Jugendlichen keine Referenzdaten in Deutschland verfügbar sind, beziehen sich alle nachfolgenden Ausführungen auf den BMI.

Anders als bei Erwachsenen, bei denen sich die epidemiologische Definition von Übergewicht und Adipositas an fixen Grenzwerten festmacht (Adipositas: BMI >30, Übergewicht: BMI >25), die einen Zusammenhang zu Krankheitsund Mortalitätsrisiken aufweisen, sind die Grenzen bei Kindern und Jugendlichen aufgrund der Dynamik ihrer körperlichen Entwicklung altersabhängig. Daher greift man zur rein statistischen Definition: Bezogen auf eine Referenzpopulation werden diejenigen Kinder oder Jugendlichen als übergewichtig beziehungsweise adipös bezeichnet, deren BMI oberhalb eines gewissen alters- und geschlechtsspezifischen Perzentils zu finden ist. So wird etwa im Referenzsystem von Kromeyer-Hauschild et al. [3] das 9o. Perzentil zur Definition von Übergewicht und das 97. Perzentil zur Definition von Adipositas verwendet. International existieren auch andere statistische Festlegungen der Grenzwerte, etwa die Definition über diejenigen Perzentile, die im Alter von 18 Jahren den BMIGrenzwerten für Erwachsene entsprechen [11]. Neben den verwendeten Perzentilen sind auch die zugrunde gelegten Referenzpopulationen unterschiedlich: Sie 
stammen aus unterschiedlichen Ländern und wurden zu unterschiedlichen Zeitpunkten erhoben. Es kann also durchaus passieren, dass ein Kind nach einem Referenzsystem normalgewichtig, nach einem anderen wiederum übergewichtig ist. Die Frage, welche BMI-Grenzwerte im Kindes- und Jugendalter am besten das Auftreten manifester Erkrankungen vorhersagen, wird sich letztlich nur durch Langzeitstudien klären lassen.

\section{Referenzsysteme zur Bestimmung der Adipositas- und Überge- wichtsprävalenz bei Kindern und Jugendlichen in Deutschland}

In Deutschland wird von der AGA (Arbeitsgemeinschaft Adipositas im Kindes- und Jugendalter, http://www.a-ga.de/aga_content.html) das Referenzsystem von Kromeyer-Hauschild et al. [3] für die Einschätzung von Übergewicht und Adipositas empfohlen, das auf einem gepoolten Datensatz von BMI-Werten von 32.422 Kindern und Jugendlichen im Alter von o bis 18 Jahren basiert. Die Daten stammen aus 17 verschiedenen Studien aus unterschiedlichen Erhebungsregionen, die zu unterschiedlichen Zeiten zwischen 1985 und 1999 durchgeführt wurden. Als Erhebungsmethoden kamen hier sowohl Messungen als auch erfragte Werte zu Größe und Gewicht zum Einsatz. Aus den Daten wurden mit der LMS-Methode [12], die auch für alle anderen hier dargestellten Referenzsysteme verwendet wurde, geglättete Perzentilkurven bestimmt. Die so bestimmten 90. beziehungsweise 97. alters- und geschlechtsspezifischen Perzentile liefern die BMI-Grenzwerte zur Definition von Übergewicht und Adipositas. Dieses Referenzsystem von Kromeyer-Hauschild et al. hat nicht so sehr den Anspruch, repräsentativ für die BMI-Verteilung der Kinder und Jugendlichen in Deutschland in einem bestimmten Zeitraum zu sein. Es dient vielmehr als „Koordinatensystem“ für die Einordnung anderer Gruppen von Kindern und Jugendlichen hinsichtlich ihres Anteils an übergewichtigen beziehungsweise adipösen Kindern. Die Tatsache beispielsweise, dass in einer Population mehr als 3\% der Kinder und Jugendlichen einen BMI oberhalb des 97. Perzen- tils haben, bedeutet in diesem Falle, dass der Anteil der Adipösen im Vergleich zum Datensatz von Kromeyer-Hauschild erhöht ist. Auf dem Vergleich mit diesem Referenzsystem beruhen in Deutschland die meisten Prävalenzaussagen zu Übergewicht und Adipositas bei Kindern und Jugendlichen.

Das etwas früher entwickelte RollandCachera-System [13], das die Gewichtsund Größendaten französischer Kinder zwischen 1953 und 1979 als Referenz verwendet und ebenfalls das 9o. beziehungsweise 97. Perzentil zur Definition von Übergewicht und Adipositas einsetzt, findet in deutschen Studien ebenfalls noch Anwendung. Dieses Referenzsystem wurde von der European Childhood Obesity Group Mitte der 1980er-Jahre (wohl auch mangels anderer Referenzsysteme) als europäischer Standard empfohlen [5]. Heute wird dieses Referenzsystem vorrangig nur noch in Frankreich und in der Schweiz verwendet.

Das von der IOTF (International Obesity Task Force) entwickelte Referenzsystem [11] basiert auf jeweils repräsentativen Daten aus sechs Ländern, die zwischen 1993 und 1999 von insgesamt etwa 200.00o Kindern, Jugendlichen und jungen Erwachsenen zwischen o und 25 Jahren erhoben wurden. Die Perzentil-Grenzwerte für Übergewicht und Adipositas sind hierbei als die Perzentile definiert, die für die Probanden im Alter von 18 Jahren zusammenfallen mit den Erwachsenengrenzwerten von 25 beziehungsweise 30. Das entspricht in den sechs beteiligten Ländern dem 83,5. bis 95,3. Perzentil als Grenzwert für Übergewicht, für Adipositas dem 96. bis 99,9. Perzentil. Für internationale Vergleiche werden deutsche Erhebungsdaten häufig auf dieses Referenzsystem bezogen.

Die Weltgesundheitsorganisation (WHO) hat für unter Fünfjährige in den Jahren von 1997 bis 2003 eine Wachstumsstudie in sechs Städten auf verschiedenen Kontinenten durchgeführt und darauf basierend einen neuen, normativ intendierten Wachstumsstandard entwickelt [14], der unter anderem Referenzkurven für den BMI liefert. Die Studie umfasste 8440 Kinder in einem Längsschnittsund einem Querschnittsteil, die restriktive Einschlussbedingungen erfüllten und unter optimalen Bedingungen aufwuchsen. Für Kinder von fünf bis 19 Jahren empfiehlt die WHO ein Referenzsystem, das auf US-amerikanischen Daten aus den Jahren von 1963 bis 1974 beruht [15]. Für die unter Fünfjährigen schlägt die WHO einen konservativen Zugang bei der Definition von Übergewicht und Adipositas vor, das heißt die Verwendung des 97,7. und 99,9. Perzentils. Für die älteren Kinder und Jugendlichen werden dagegen das 84,1. und 97,7. Perzentil zur Definition von Übergewicht und Adipositas herangezogen (M. de Onis, persönliche Mitteilung), die wie die IOTF-Grenzwerte im Alter von 18 Jahren mit den Erwachsenengrenzwerten übereinstimmen.

Ein weiteres mögliches neues Referenzsystem für Kinder und Jugendliche in Deutschland lässt sich mithilfe der aktuellen repräsentativen Daten des Kinder- und Jugendgesundheitssurveys (KiGGS) etablieren: Die für 8645 Jungen und 8378 Mädchen $^{1}$ zwischen o und 18 Jahren standardisiert erhobenen BMI-Werte (siehe auch nächster Abschnitt) wurden ebenfalls nach der Methodik von Cole [12] zu Referenzkurven verarbeitet und in [16] veröffentlicht. Besondere Sorgfalt wurde dabei auf die Modellierung des Kurvenverlaufs in den ersten Lebensjahren verwendet, sodass diese Kurven auch für unter Zweijährige anwendbar sind. Dieses Referenzsystem steht im Einklang mit den internationalen Entwicklungen und überwindet die methodischen Einschränkungen der Datenbasis von KromeyerHauschild.

Es existieren weitere nationale und internationale Referenzsysteme (siehe die Arbeit von Pigeot et al. in diesem Heft [17]), die allerdings bislang bei der Bewertung deutscher Daten kaum eine Rolle spielten.

\section{Erhebungsmethoden}

Über die Wahl des zugrunde gelegten Referenzsystems hinaus ist methodisch weiterhin von Bedeutung, auf welche Art und Weise der BMI ermittelt wurde. Der BMI, der auf der standardisierten Messung von

\footnotetext{
1 Kinder mit relevanten Begleiterkrankungen/ Medikationen wurden aus der Referenzpopulation ausgeschlossen.
} 
Bundesgesundheitsbl 2010 · 53:643-652 DOI 10.1007/s00103-010-1083-2

(c) Springer-Verlag 2010

\section{B.-M. Kurth · A. Schaffrath Rosario Übergewicht und Adipositas bei Kindern und Jugendlichen in Deutschland}

Zusammenfassung

Die Häufigkeit von Übergewicht und Adipositas bei Kindern und Jugendlichen ist in den letzten Jahren auch in Deutschland auf besorgniserregende Weise angestiegen. Dabei gibt es unterschiedliche Methoden und Datenquellen, um das Ausmaß dieses PublicHealth-Problems abzuschätzen. Die vorliegende Arbeit gibt einen Überblick über die für Deutschland relevanten Referenzsysteme für den aus Körpergröße und Körpergewicht bestimmten Body-Mass-Index (BMI) und über die vorliegenden Datenquellen: die Schuleingangsuntersuchungen, die HBSC-Studie der WHO und den Kinder- und Jugendgesundheitssurvey KiGGS. Aktuelle Häufigkeitsschätzungen basieren auf den KiGGS-Daten, der unter Zugrundelegung des Referenzsystems von Kromeyer-Hauschild ermittelt hat, dass in Deutschland insgesamt 14,8\% der Kinder und Jugendlichen im Alter von zwei bis 17 Jahren übergewichtig sind, davon lei- den 6,1\% unter Adipositas. Die Hochrechnung dieser Prozentzahlen auf absolute Werte ergibt, bezogen auf den aktuellen Bevölkerungsstand, dass in Deutschland zirka 1,7 Millionen übergewichtige Kinder und Jugendliche im Alter ab zwei Jahren leben, wovon 750.000 adipös sind. Dieser Status quo stellt die Messlatte für alle künftigen Erhebungen des BMI dar mit dem Ziel, zeitliche Trends zu bestimmen und damit die Wirksamkeit nationaler Präventions- und Interventionsprogramme zu überprüfen. Die für bestimmte Altersgruppen der Kinder und Jugendlichen in Deutschland regelmäßig durchgeführten Erhebungen von Körpergröße und -gewicht wie beispielsweise im Rahmen der Schuleingangsuntersuchungen sind bei der Einhaltung von Erhebungsstandards geeignet, solche zeitlichen Trends abzuschätzen. Erhebungen, die anstelle von Messwerten auf erfragten Angaben zu Körpergröße und -ge- wicht beruhen, wie der deutsche Teil der HBSC-Studie, bedürfen dabei jedoch einer Korrektur dieser Angaben anhand der subjektiven Einschätzung des Körperbildes. Auch die KiGGS-Probanden werden in größeren Abständen erneut untersucht und befragt. Damit kann die Einschätzung der Verbreitung von Übergewicht und Adipositas regelmäBig aktualisiert werden. Das bestehende Referenzsystem von Kromeyer-Hauschild sollte dabei jedoch nicht durch die KiGGS-BMI-Werte ersetzt werden, um die Angaben zur Häufigkeit von Übergewicht und Adipositas nicht künstlich herunterzurechnen. Es sollte jedoch nicht für Kinder unter zwei Jahren verwendet werden.

Schlüsselwörter Body-Mass-Index - Übergewicht und Adipositas · Prävalenz $\cdot$ Kinder und Jugendliche $\cdot$ Deutschland

\section{Overweight and obesity in children and adolescents in Germany}

\section{Abstract}

The increasing prevalence of overweight and obesity is a reason for concern not only in Germany but also in other countries. There are various methods and data sources that can be used to assess the extent of this public health problem. The present publication gives an overview of the reference systems that are in use in Germany to assess body mass index (BMI), which is calculated from height and weight, and an overview from several data sources: the school entry examinations, the HBSC study of the WHO, and the German Health Interview and Examination Survey for Children and Adolescents (KiGGS). Current prevalence estimates are based on the KiGGS survey, which found that, based on the Kromeyer-Hauschild reference system, $14.8 \%$ of the children and adolescents in Germany aged 2-17 years are overweight, including $6.1 \%$ suffering from obesity. In absolute numbers referring to the most recent population numbers, this corresponds to 1.7 million overweight children and adolescents in Germany aged 2 years and older, 750,000 of whom are obese. This description of the status quo represents the benchmark for all future studies of BMI that aim at assessing temporal trends and, thus, the efficacy of national prevention and intervention programs. Regular examinations of certain age groups of children and adolescents in Germany, such as the school entry examinations, can be used for the assessment of temporal trends, if certain standards are followed during the examination. Studies that rely on self-reported height and weight instead of measurements, such as the German part of the HBSC study, however, need a correction based on the subjective body perception. The KiGGS participants themselves are also subject to followup interviews and examinations. Thus, it will be possible to regularly update the assessment of the prevalence of overweight and obesity. The Kromeyer-Hauschild reference system should not be replaced by the KiGGS BMI data, in order not to artificially lower the prevalences of overweight and obesity. It should, however, not be used for children below 2 years of age.

\section{Keywords}

Body mass index - Overweight and obesity . Prevalence - Children and adolescents . Germany 
Größe und Gewicht beruht, hat einen anderen Stellenwert als der, der auf den subjektiven Angaben der Kinder und Jugendlichen beziehungsweise deren Eltern beruht. Es ist aus vergleichenden Studien bekannt, dass die subjektive Bestimmung des BMI zu einer großen Unterschätzung der Auftretenshäufigkeit von Übergewicht und Adipositas führt [18]. Dies muss bei regionalen, zeitlichen und sozialspezifischen Vergleichen unterschiedlicher Studienergebnisse Berücksichtigung finden. Aus der Literatur sind verschiedene Korrekturverfahren bekannt, die aber jeweils studienspezifisch und damit kaum übertragbar sind. Bei der Feststellung zeitlicher Trends und regionaler Unterschiede auf nationaler Ebene ist anzustreben, dass Studienergebnisse verglichen werden, die auf identischen Erhebungsmethoden beruhen.

\section{Datengrundlagen}

Zur Einschätzung von Übergewicht und Adipositas gibt es in Deutschland in den letzten Jahrzehnten unterschiedliche Datenquellen. Nicht alle davon können den Anspruch erheben, repräsentativ zu sein. Von genereller Bedeutung für die Epidemiologie von Übergewicht und Adipositas in Deutschland sind regelmäßig durchgeführte Erhebungen mit vergleichbarer Methodik und definierter Grundpopulation.

Schuleingangsuntersuchungen. Zu diesen Datenquellen gehören zum einen die jährlich in allen Bundesländern durchgeführten Schuleingangsuntersuchungen, bei denen die Kinder auch gemessen und gewogen werden. Die Erfassung des BMI von Kindern und Jugendlichen im Alter zwischen fünf und sechs Jahren ermöglicht theoretisch sehr genaue Prävalenzschätzungen für die entsprechenden $\mathrm{Ge}$ burtsjahrgänge. Da die Schuleingangsuntersuchungen zum Ziel haben, die individuelle Schultauglichkeit eines Kindes zu prüfen, erfüllen sie jedoch nicht automatisch die Ansprüche einer epidemiologischen Studie: Es ist schwierig, die für epidemiologische Auswertungen erforderliche Standardisierung der Messungen abzusichern. Der aus epidemiologischer Sicht nachvollziehbare Wunsch, dass Körpergröße und -gewicht unter vergleichbaren Bedingungen (mit oder ohne Schuhe, mit oder ohne Bekleidung, in gleicher Haltung, mit gleichem Messgerät) erhoben werden, gewinnt aber im Zusammenhang mit der Gesundheitsberichterstattung zunehmend an Akzeptanz. Hier ist der Öffentliche Gesundheitsdienst (ÖGD), der in den meisten Bundesländern sowohl für die Schuleingangsuntersuchungen als auch für die Gesundheitsberichterstattung bei Kindern und Jugendlichen zuständig ist, zunehmend und erfolgreich um eine Vereinheitlichung der Methoden bemüht. Das macht nicht nur die jährlichen Aussagen zuverlässiger und besser vergleichbar, sondern erweitert die Möglichkeiten von Trendaussagen erheblich.

HBSC-Studie. Eine weitere Datenquelle ist die von der WHO seit 1993 im Abstand von vier Jahren durchgeführte multizentrische HBSC-Studie (Health Behaviour in School-aged Children). Die Zielpopulation dieser Studie sind Schulkinder aus den fünften, siebten und neunten Klassen [19]. Die Kinder und Jugendlichen werden in dieser Studie allerdings nicht gemessen und gewogen, sondern nach Größe und Gewicht gefragt. Diese subjektiven Angaben sind zu unterscheiden von den objektiven Messungen. An der Studie beteiligen sich mehr als 40 Länder aus Europa, Nordamerika und Israel. Die deutsche Stichprobe resultiert jeweils aus einer Zufallsstichprobe von Schulklassen aller öffentlichen Schulen der fünften, siebten und neunten Klassenstufe. Die hier dargestellten Ergebnisse stammen aus den Jahren 2005/2006 und umfassen die Bundesländer Nordrhein-Westfalen, Berlin, Hessen und Sachsen [20, 21]. Um annähernde Repräsentativität zu erreichen, wird für Deutschland jeweils ein „strukturtypischer Datensatz“ konstruiert [21]. In der HBSC-Studie werden subjektive Angaben der Schüler zu Körpergröße und -gewicht erhoben, um über die BMIBerechnung Prävalenzaussagen zu Übergewicht und Adipositas für die entsprechenden Altersgruppen in verschiedenen europäischen Ländern zu treffen.

KiGGS. Der Kinder- und Jugend-Gesundheitssurvey (KiGGS) liefert als repräsenta- tive Gesundheitsstudie für Kinder und Jugendliche in Deutschland die derzeit beste und aktuellste Datenbasis für Prävalenzaussagen zu Übergewicht und Adipositas bei Kindern und Jugendlichen in Deutschland: Die Studie wurde von Mai 2003 bis Mai 2006 durchgeführt, es nahmen insgesamt 17.641 Kinder und Jugendliche im Alter von o bis 17 Jahren gemeinsam mit ihren Eltern teil [22]. KörpergröBe und -gewicht wurden in standardisierter Weise gemessen, wobei dies bei den jüngeren Kindern je nach Entwicklungsstand entweder im Liegen oder auch im Stehen durchgeführt wurde. Ab dem $\mathrm{Al}-$ ter von zwei Jahren wurde die Körpergröße generell im Stehen, ohne Schuhe mit einem kalibrierten Stadiometer auf o, $1 \mathrm{~cm}$ genau gemessen. Das Gewicht wurde in Unterwäsche auf einer kalibrierten Waage auf $0,1 \mathrm{~kg}$ genau abgelesen (genauere Ausführungen hierzu in [2]). Der Sozialstatus der Familien wurde anhand von Bildungsstand, beruflicher Stellung und Einkommen der Eltern ermittelt [23]. Als Migranten zählen Kinder, die selbst oder ein Elternteil von ihnen im Ausland geboren sind und/oder eine nicht-deutsche Staatsangehörigkeit besitzen [24]. Alle hier dargestellten KiGGS-Prävalenzen wurden gewichtet berechnet [25]. Standardfehler wurden mit der SAS 9.2-SURVEYFREQProzedur [26] unter Berücksichtigung der Clusterung der Kinder innerhalb der gezogenen Gemeinden berechnet und daraus unter Nutzung der Logit-Transformation 95\%-Konfidenzintervalle für die Prävalenzen gebildet.

Im Rahmen des Gesundheitsmonitorings am Robert Koch-Institut werden die KiGGS-Teilnehmer in regelmäßigen Abständen erneut befragt und untersucht (Etablierung einer KiGGS-Kohorte [27]). Im Jahr 2009 wurde eine telefonische Nachbefragung der KiGGS-Probanden begonnen, die 2011 abgeschlossen sein soll.

Ab 2012 sollen die KiGGS-Probanden wiederum in Studienzentren untersucht und befragt werden. Die Stichprobe soll dann jeweils so aufgestockt werden, dass für jede Altersgruppe zwischen o und 18 Jahren bei jeder Welle repräsentative Aussagen getroffen werden können. Dies bedeutet zum einen, dass im Abstand von vier bis fünf Jahren repräsentative 
Querschnittsaussagen zur Prävalenz von Übergewicht und Adipositas getroffen werden können. Darüber hinaus wird es aber möglich sein, die BMI-Entwicklung im Lebensverlauf zu beobachten und damit repräsentative epidemiologische Verlaufsdaten zu erhalten.

\section{Aktuelle Ergebnisse}

\section{KiGGS}

Die KiGGS-Ergebnisse zur Auftretenshäufigkeit von Übergewicht und Adipositas beruhen auf den BMI-Kategorisierungen nach dem Referenzsystem von Kromeyer-Hauschild et al. [3]. Bislang wurden von uns keine Prävalenzschätzungen für unter Dreijährige angegeben. Dies lag daran, dass sich bei Verwendung dieses Referenzsystems für unter Dreijährige unerwartet hohe Prävalenzen ergeben. Hierfür kamen drei mögliche Ursachen in Betracht:

1. Verzerrungen aufgrund der Verwendung von in Sechsmonats-Abständen tabellierten Referenzwerten,

2. Verzerrungen, die sich durch die Tatsache ergeben, dass KiGGS-Kinder im zweiten Lebensjahr nicht immer im Liegen gemessen wurden, sondern je nach Entwicklungsstand auch im Stehen, was zu o,5 bis o, $8 \mathrm{~cm}$ niedrigeren Größenangaben und damit höheren BMI-Werten führt $[28,29]$,

3. Verzerrungen, die in den Referenzdaten begründet sind.

Ursache (1) und (2) können wir jedoch mittlerweile ausschließen, da sich die Prävalenzen bei Verwendung monatsweiser Referenzwerte nach Kromeyer-Hauschild et al. nicht wesentlich verändern und außerdem die Prävalenzen im ersten Lebensjahr, in dem Messungen im Stehen noch nicht auftraten, deutlich höher liegen als im zweiten. Als wahrscheinlichste Ursache für die erhöhten Prävalenzen sehen wir daher Verzerrungen in den Referenzwerten selbst, die dadurch zustande kommen, dass eine Modellierung von BMI-Perzentilkurven über den gesamten Altersbereich von o bis 17 Jahren den starken Veränderungen des BMI in den ersten beiden Lebensjahren nicht gerecht werden kann [16]. Diese Verzerrungen kön-

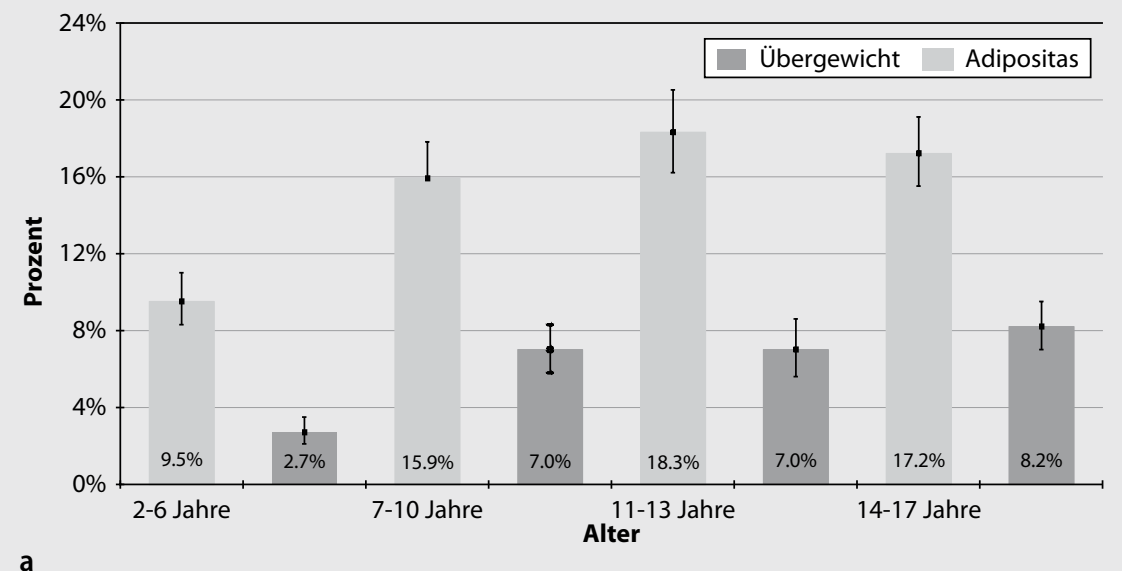

a

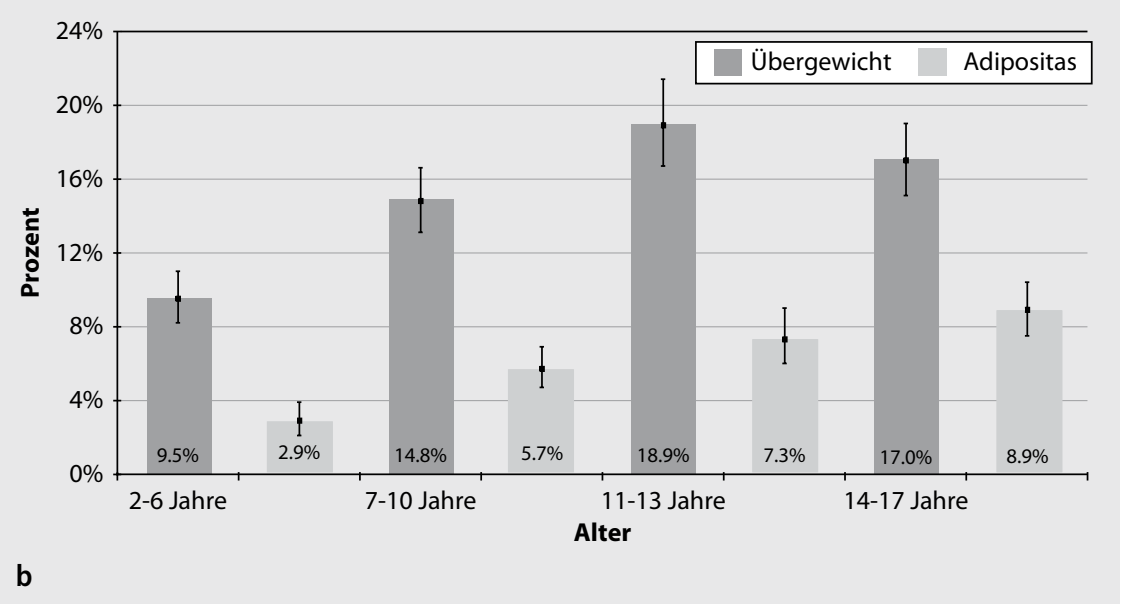

Abb. $1 \Delta$ a Übergewicht und Adipositas bei Jungen in KiGGS (2003 bis 2006) nach Altersgruppen (Referenzsystem: Kromeyer-Hauschild [3]). b Übergewicht und Adipositas bei Mädchen in KiGGS (2003 bis 2006) nach Altersgruppen (Referenzsystem: Kromeyer-Hauschild [3])

nen noch dadurch verstärkt worden sein, dass die Kromeyer-Hauschild-Daten für Kleinkinder auf Vorsorgeuntersuchungen beruhen, also nur BMI-Werte für ausgewählte Zeitpunkte im Altersverlauf enthalten.

$\mathrm{Ab}$ dem Alter von zwei Jahren scheinen die Daten von Kromeyer-Hauschild jedoch weiterhin zur Beurteilung der Prävalenz von Übergewicht und Adipositas in Deutschland geeignet zu sein, sodass hier die Prävalenzen nach dem Kromeyer-Hauschild-Referenzsystem [3] für die Altersgruppe zwei bis 17 Jahre (7998 Jungen und 7664 Mädchen) dargestellt werden. Für „unter Dreijährige“ verzichten wir auf Prävalenzaussagen nach Kromeyer-Hauschild.

Danach sind in Deutschland insgesamt $14,8 \%$ der Kinder und Jugendlichen im Alter von zwei bis 17 Jahren übergewichtig, davon leiden 6,1\% unter Adipositas. Die Hochrechnung dieser Prozentzahlen auf absolute Werte ergibt bezogen auf den aktuellsten verfügbaren Bevölkerungsstand vom 31.12.2008 [30], dass in Deutschland zurzeit zirka 1,7 Millionen übergewichtige Kinder und Jugendliche im Alter ab zwei Jahren leben, von diesen sind 750.000 adipös. (Diese Zahlen beruhen auf der Annahme, dass sich die Prävalenzen im Vergleich zum Zeitraum 2003 bis 2006 nicht verändert haben.) Die Anpassung an den aktuellen Bevölkerungsstand führt aufgrund der abnehmenden Zahl von Kindern in der Bevölkerung zu einer etwas geringeren Absolutzahl als in [2] für den Bevölkerungsstand 31.12.2004 berichtet.

Die Ergebnisse von KiGGS ergeben im Vergleich zur Gruppe der zwei- bis 17-jährigen Kinder und Jugendlichen, die als Referenzpopulation für Kromeyer-Hauschild et al. [3] in den achtziger und neunziger Jahren dienten, eine Steigerungsrate der Übergewichtigen um etwa 50\%; bei den Adipösen hat eine Verdoppelung stattgefunden. Diese sehr gro- 


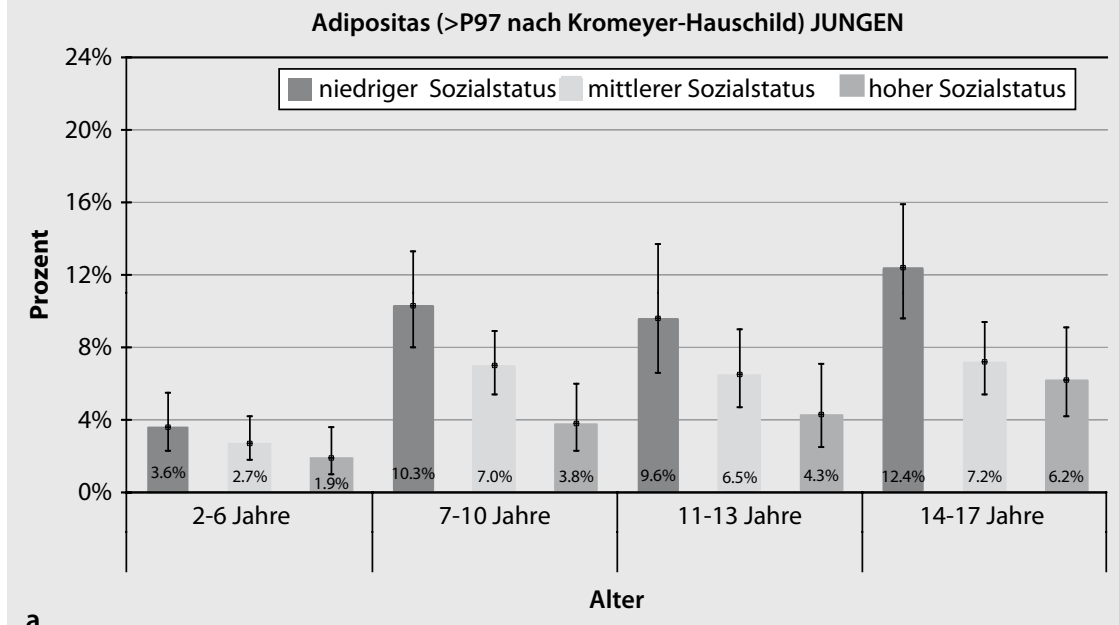

a

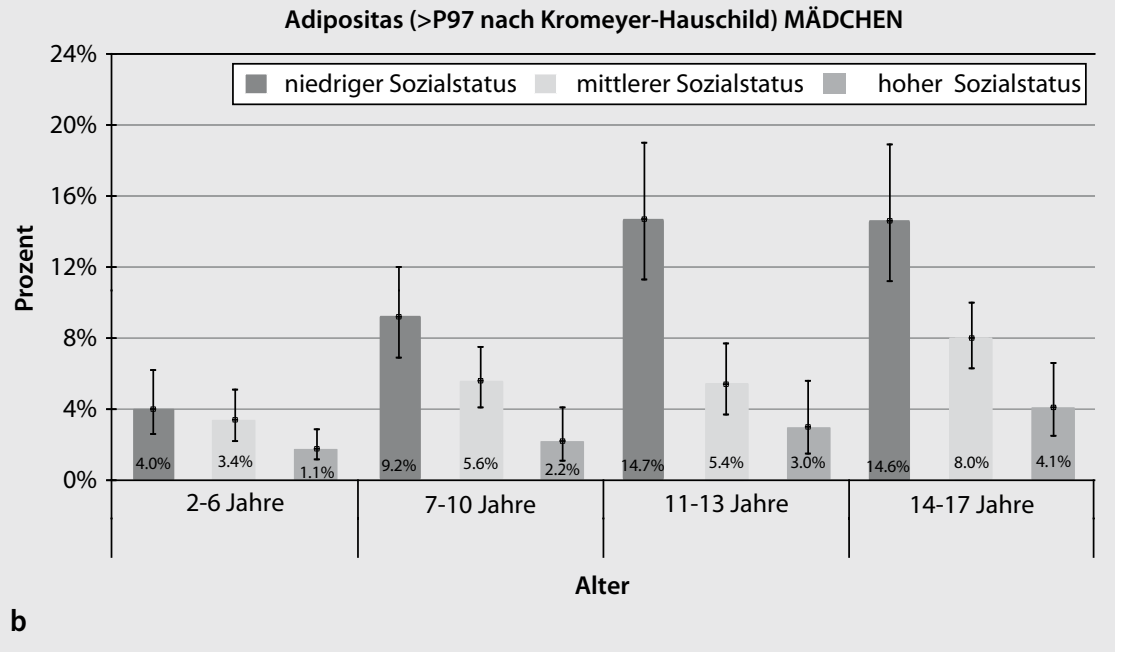

Abb. $2 \Delta$ a Adipositas bei Jungen in KiGGS (2003 bis 2006) nach Altersgruppen und Sozialstatus (Referenzsystem: Kromeyer-Hauschild [3]). b Adipositas bei Mädchen in KiGGS (2003 bis 2006) nach Altersgruppen und Sozialstatus (Referenzsystem: Kromeyer-Hauschild [3])

ben Prävalenzaussagen differenzieren sich nach Alter, aber kaum nach Geschlecht (• Abb. 1a, b).

Für Jungen und Mädchen zusammengenommen steigt der Anteil der Übergewichtigen von $10 \%$ bei den Zwei- bis Sechsjährigen über $15 \%$ bei den Siebenbis Zehnjährigen bis hin zu $17 \%$ bei den 14- bis 17-Jährigen. Die Verbreitung von Adipositas liegt bei den Zwei- bis Sechsjährigen bei $2,8 \%$ und steigt über $6,4 \%$ bei den Sieben- bis Zehnjährigen bis auf 8,5\% bei den 14- bis 17-Jährigen.

Bei einer zusätzlichen Differenzierung nach Sozialstatus [23] ergeben sich die in - Abb. 2a und b für die Adipositas dargestellten Verhältnisse. Kinder und Jugendliche aus sozial benachteiligten Familien haben ein höheres Risiko für Übergewicht und Adipositas. Dies zeigt sich extrem bei Mädchen im Alter von 14 bis
17 Jahren aus Familien mit niedrigem Sozialstatus: Hier beträgt die Adipositasrate fast das Fünffache des als ,normal“ anzusehenden Wertes. Ähnliche Verhältnisse finden sich auch für Jungen des entsprechenden Alters aus sozial benachteiligten Familien. tionshintergrund [24] ergibt wiederum einen erhöhten Anteil an übergewichtigen und adipösen Kindern und Jugendlichen aus Migrantenfamilien ( $\bullet$ Abb. 3a und b für Adipositas). Allerdings differenziert sich das sehr nach der Nationalität: Wie in [31] dargestellt, sind Mädchen und Jungen aus der Türkei, Mädchen aus Mittelund Südeuropa sowie Jungen aus Polen am häufigsten von Übergewicht betroffen, während Kinder von Aussiedlern aus der ehemaligen Sowjetunion sogar leicht geringere Raten aufweisen als die deutschen
Eine Differenzierung nach dem Migra-
Kinder ohne Migrationshintergrund. Mit längerer Aufenthaltsdauer in Deutschland steigen die Prävalenzen aber auch bei diesen Migranten an.

Als regionale Differenzierung mit dem Anspruch auf Repräsentativität lässt der KiGGS lediglich einen Ost-West-Vergleich zu. Die alters- und geschlechtsspezifischen Adipositas- und Übergewichtsraten weisen keine eindeutigen signifikanten Unterschiede zwischen den alten und neuen Bundesländern auf [2].

Mithilfe des KiGGS-Datensatzes, der wesentliche Komponenten des Gesundheitsverhaltens der Kinder und Jugendlichen selbst sowie des sozialen Umfelds umfasst, lassen sich mögliche Einflussfaktoren auf Übergewicht und Adipositas in der gesamten Komplexität ihres $\mathrm{Zu}$ sammenwirkens bestimmen. Ergebnisse dieser Analysen finden sich in [32]. Als wichtigste Prädiktoren haben sich dabei ein niedriger Sozialstatus und das Übergewicht der Eltern erwiesen. Darüber hinaus wurden in einem multivariaten Modell statistisch signifikante Assoziationen mit dem Rauchen der Mutter während der Schwangerschaft, einem hohen Geburtsgewicht und einer hohen Gewichtszunahme während der Schwangerschaft sowie einem hohen Medienkonsum gefunden. Eine lange Schlafdauer hatte einen protektiven Effekt.

\section{Schuleingangsuntersuchungen}

In der Publikation von Moß et al. 2007 [33] werden die Prävalenzaussagen aus den Schuleingangsuntersuchungen der einzelnen Länder in den Jahren 2003/2004 zusammengetragen und verglichen. Als Grundlage dient auch hier das Referenzsystem von Kromeyer-Hauschild et al. [3]. Auch wenn die Angaben zu Übergewicht und Adipositas sehr uneinheitlich dokumentiert sind, lassen sich erhebliche regionale Unterschiede feststellen. Die Befundhäufigkeit für Übergewicht bei den Fünf- bis Sechsjährigen liegt zwischen $7,2 \%$ in Thüringen und 13,6\% in Mecklenburg-Vorpommern. Für Adipositas finden sich Prävalenzen zwischen 3,6\% in Bayern und 6,1\% in Mecklenburg. Es lässt sich zusammenfassend ein Nord-Süd-Gefälle, aber kein Ost-West-Unterschied feststellen. Zum 
Vergleich mit den Schuleingangsuntersuchungen sind in $\bullet$ Tab. 1 die entsprechenden Prävalenzen in den KiGGS-Daten angegeben. Da die Höhe der Prävalenzen auch vom genauen Alter der Kinder bei der Schuleingangsuntersuchung abhängt, sind sie in $\bullet$ Tab. 1 für verschiedene Altersbereiche ausgewiesen (siehe dazu auch $[34,35]$ ).

Mit den regelmäßig durchgeführten Schuleingangsuntersuchungen lassen sich zeitliche Trends in der Entwicklung der Übergewichts- und Adipositasraten nachweisen. Wie ebenfalls in [33] festgestellt, ist seit Beginn der 199oer-Jahre ein kontinuierlicher Anstieg des Anteils übergewichtiger und adipöser Einschüler festzustellen. Dies wird in [36] sowohl für ostals auch für westdeutsche Kinder, ebenfalls auf Basis der Schuleingangsuntersuchungen in den alten und neuen Bundesländern, bestätigt. Die in einigen Bundesländern festzustellende Stagnation des Anstiegs gibt derzeit Anlass zu der Hypothese, dass die Entwicklung der Adipositasprävalenz ihren Höhepunkt erreicht hat und künftig eine leicht rückläufige Tendenz aufweisen könnte.

\section{HBSC-Studie}

Die deutsche HBSC-Studie 2005/2006, die fast zeitgleich mit dem KiGGS durchgeführt wurde, hat 11.513 Schülerinnen und Schüler aus den beteiligten Bundesländern einbezogen. Der strukturtypische Datensatz für Deutschland, so wie er auch in den internationalen Datensatz eingeflossen ist, umfasst die Angaben von 7274 Kindern und Jugendlichen aus der fünften, siebten und neunten Klasse. Deren Angaben zu Größe und Gewicht ergaben über die BMI-Berechnung und bezogen auf das Referenzsystem von Kromeyer-Hauschild [3] die in $\bullet$ Tab. 2 dargestellten Prävalenzen von Übergewicht und Adipositas [37].

In einem internationalen Vergleich der Adipositas- und Übergewichtsraten aller an der HBSC-Studie beteiligten Länder wurde das IOTF-Referenzsystem zugrunde gelegt [38]. Danach ergaben sich für Deutschland 14,5\% übergewichtige Jungen, von diesen waren 2,4\% adipös, und 9,4\% übergewichtige Mädchen, davon $1,5 \%$ adipös.

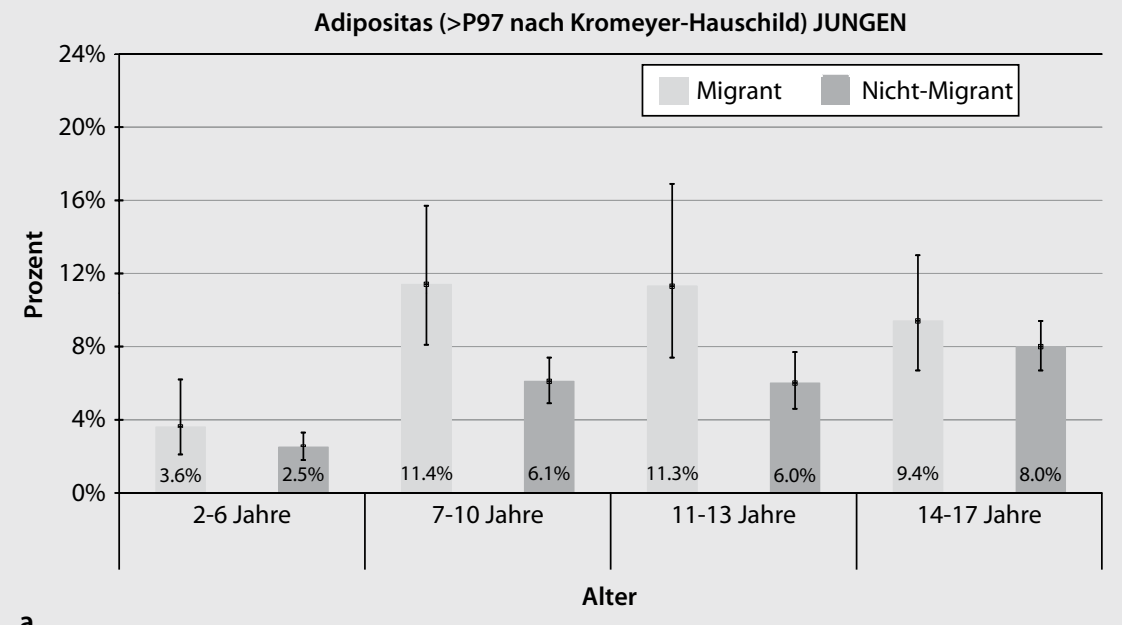

a

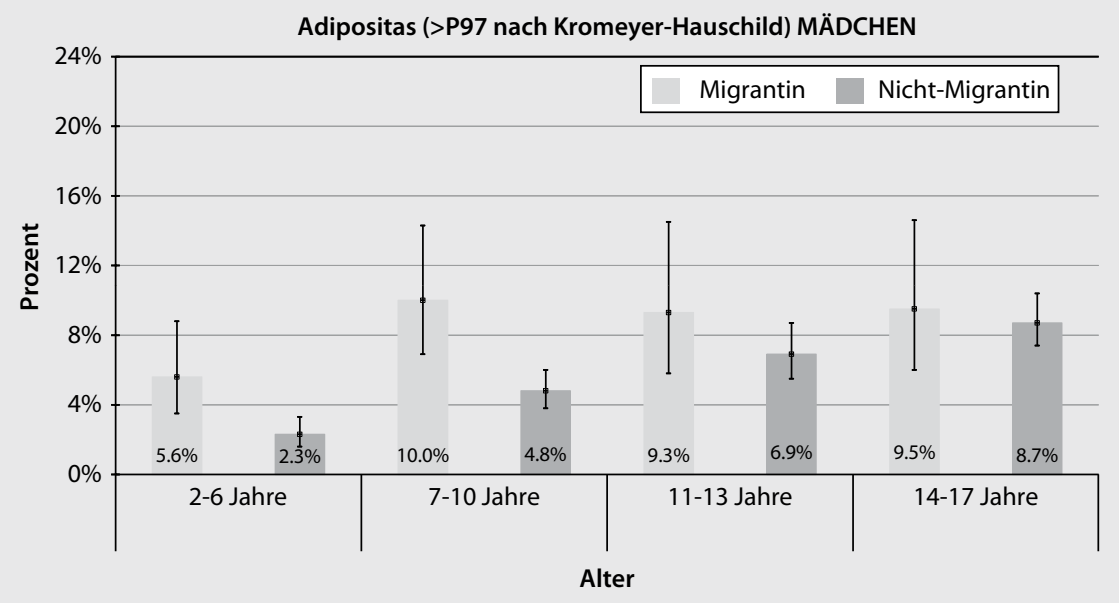

b

Abb. $3 \Delta$ a Adipositas bei Jungen in KiGGS (2003 bis 2006) nach Altersgruppen und Migrationshintergrund (Referenzsystem: Kromeyer-Hauschild [3]). b Adipositas bei Mädchen in KiGGS (2003 bis 2006) nach Altersgruppen und Migrationshintergrund (Referenzsystem: Kromeyer-Hauschild [3])

Der Vergleich der HBSC-Ergebnisse mit den ebenfalls in $\bullet$ Tab. 2 enthaltenen KiGGS-Daten für die entsprechenden Klassenstufen zeigt, dass die HBSC-Studie für beide Referenzsysteme zu deutlich niedrigeren Prävalenzschätzungen kommt als KiGGS.

\section{KiGGS-Ergebnisse mit anderen Referenzsystemen}

Geht man davon aus, dass die besten zurzeit verfügbaren Daten zum Auftreten von Übergewicht und Adipositas in ganz Deutschland die KiGGS-Daten sind, so sind es auch diese, die in internationale Vergleiche Eingang finden sollten. Das bedingt jedoch, wie eingangs erläutert, dass die Berechnung der Prävalenzen für den KiGGS auch für die internationalen Referenzsysteme erfolgt. Die Ergebnisse sind in 0 Tab. 3 dargestellt. Sowohl nach dem IOTFReferenzsystem als auch nach dem der WHO (mit altersspezifischen Definitionen von Übergewicht und Adipositas) ist die Prävalenz von Übergewicht höher als nach dem Referenzsystem von Kromeyer-Hauschild. Bei der Adipositas liegen die IOTF-Prävalenzen niedriger, die WHO-Prävalenzen höher (bei Jungen) beziehungsweise gleich hoch (bei Mädchen). Die höchsten Prävalenzen, vor allem für Adipositas, ergeben sich nach der Referenz von Rolland-Cachera. Nach den WHO-Referenzen liegen die Prävalenzen bei Jungen etwas höher als bei Mädchen. Detaillierte Angaben und eine ausführlichere Diskussion sind einer demnächst erscheinenden Publikation der beiden Autorinnen vorbehalten. 
Tab. 1 Häufigkeit von Übergewicht und Adipositas in KiGGS (2003 bis 2006) im Schuleingangsalter (Referenzsystem: Kromeyer-Hauschild [3]) (vgl. [34])

\begin{tabular}{|c|c|c|c|}
\hline Altersbereich $^{\mathrm{a}}$ & 5,5 bis unter 7,0 Jahre & 5,25 bis unter 6,75 Jahre & 5,0 bis unter 6,5 Jahre \\
\hline Übergewicht ${ }^{b}$ & $9,7 \%(8,0-11,7)$ & $8,8 \%(7,2-10,7)$ & $8,8 \%(7,1-10,8)$ \\
\hline Adipositas & $3,1 \%(2,3-4,3)$ & $2,5 \%(1,7-3,6)$ & $2,8 \%(1,9-4,0)$ \\
\hline
\end{tabular}

Tab. 2 Vergleich der in der HBSC-Studie 2005 bis 2006 aus Selbstangaben ermittelten prozentualen Anteile übergewichtiger und adipöser Jugendlicher in der fünften, siebten und neunten Klasse mit den für dieselben Schulklassen berechneten Anteilen bei KiGGS (2003 bis 2006) (auf Messwerten beruhend)

\begin{tabular}{|c|c|c|c|c|c|}
\hline \multirow{2}{*}{$\begin{array}{l}\text { Referenzsys- } \\
\text { tem }\end{array}$} & & \multicolumn{2}{|l|}{ Jungen } & \multicolumn{2}{|l|}{ Mädchen } \\
\hline & & KiGGS & HBSC $^{a}$ & KiGGS & $\mathrm{HBSC}^{\mathrm{a}}$ \\
\hline \multirow{2}{*}{$\begin{array}{l}\text { Kromeyer- } \\
\text { Hauschild [3] }\end{array}$} & Übergewicht ${ }^{b}$ & $18,4 \%(16,3-20,7)$ & $9,2 \%$ & $17,9 \%(15,7-20,3)$ & $7,3 \%$ \\
\hline & Adipositas & $8,3 \%(6,9-10,1)$ & $3,0 \%$ & $7,7 \%(6,1-9,7)$ & $2,5 \%$ \\
\hline \multirow[t]{2}{*}{ IOTF [11] } & Übergewicht ${ }^{b}$ & $23,6 \%(21,1-26,2)$ & $14,5 \%$ & $21,8 \%(19,5-24,3)$ & $9,4 \%$ \\
\hline & Adipositas & $6,3 \%(5,1-7,8)$ & $2,4 \%$ & $5,7 \%(4,4-7,4)$ & $1,5 \%$ \\
\hline
\end{tabular}

Für KiGGS: Prävalenz mit 95\%-Konfidenzintervall (Logit-Intervall unter Berücksichtigung des Stichprobendesigns). ${ }^{a}$ Angaben für das Kromeyer-Hauschild-Referenzsystem aus Referenz [37], für IOTF aus [38]. bInklusive Adipositas. IOTF International Obesity Task force.

\section{Diskussion}

Die KiGGS-Ergebnisse geben eine präzise Standortbestimmung des Gesundheitszustands und Gesundheitsverhaltens der in Deutschland lebenden Kinder und Jugendlichen. Die aktuellen Daten zur Auftretenshäufigkeit von Übergewicht und Adipositas und die Identifizierung von Risikogruppen unter den Kindern und Jugendlichen belegen den Ernst der Situation. Die Analysen der Zusammenhänge und die Bestimmung möglicher Determinanten gehen weit über das hinaus, was die Schuleingangsuntersuchungen und auch die HBSC-Studie leisten können. Es ergeben sich jedoch aus KiGGS methodische Schlussfolgerungen für die beiden anderen Erhebungen:

Die weiter oben anhand der KiGGSDaten festgestellten Unterschiede zwischen Kindern mit und ohne Migrationshintergrund bei Übergewicht und Adipositas sprechen dafür, dass zum einen der Migrationshintergrund in allen Studien, die Körpergröße und Körpergewicht messen, ebenfalls erfasst werden sollte. Konsequenzen hat das beispielsweise für die Auswertung der Schuleingangsuntersuchungen in Großstädten mit einem hohen Anteil an Migrantenkindern. Hier werden die Prävalenzen von Übergewicht und Adipositas zunehmend differenziert nach Migrationshintergrund ausgewertet.
Die in [33] dargestellten regionalen Unterschiede in der Übergewichts- und Adipositasprävalenz von Einschülern auf Bundesländerebene lassen sich auch noch feinteiliger, zum Beispiel auf kommunaler Ebene, feststellen [35]. Einflussfaktoren, wie beispielsweise das Bildungsniveau der Eltern oder der Migrationshintergrund der Familien, könnten durchaus Erklärungsansätze für regionale Unterschiede liefern. Von Nachteil ist es jedoch, dass die Schuleingangsuntersuchungen nicht in allen Bundesländern die Erfassung des Sozialstatus oder zumindest des Bildungsniveaus der Eltern mit vorsehen.

In [34] weisen Rosario und Kurth nach, dass darüber hinaus die unterschiedliche Alterszusammensetzung der Gruppe der untersuchten Einschüler durchaus Erklärungen für regionale Unterschiede in der Adipositasprävalenz liefern können: Wie der in KiGGS quantifizierte Altersgang der Übergewichts- und Adipositasentwicklung nahelegt, nimmt das Risiko für Übergewicht in der Altersgruppe der Einschüler mit jedem Lebensmonat zu. Damit hat ein Bundesland, das seine Einschulungsuntersuchungen etwas später durchführt als ein anderes, allein schon aus diesem Grund schlechtere Ergebnisse zu erwarten. Ähnliche Konsequenzen hat dies auch für die Bewertung zeitlicher Trends: Die Möglichkeit, Kinder bereits im jüngeren Alter einzuschulen, erhöht langfristig den Anteil der Vier- bis Fünfjährigen, die an den Einschulungsuntersuchungen teilnehmen und könnte ein Erklärungsansatz für stagnierende Adipositasraten sein. In [35] wird dies für Nordrhein-Westfalen genau analysiert. Die Notwendigkeit, die Gruppe der Einschüler nicht mehr insgesamt, sondern altersspezifisch zu beschreiben, wird hierbei sehr offensichtlich.

Dass auch rein „handwerkliche“ Fehler wie die falsche Zuordnung der Referenzwerte des Kromeyer-Hauschild-Referenzsystems zu den entsprechenden $\mathrm{Al}$ tersgruppen der untersuchten Kinder zu Artefakten bei der Bestimmung regionaler und zeitlicher Unterschiede führen können, wird ebenfalls in [34] gezeigt.

Der Vergleich der im Rahmen der deutschen HBSC-Studie ermittelten Übergewichts- und Adipositasprävalenzen mit den fast zeitgleich erhobenen KiGGSDaten ergibt markant abweichende Aussagen. Die HBSC-Studie bildet die vergleichsweise bedrohliche Situation des Auftretens von Übergewicht und Adipositas nicht annähernd ab. Diese Abweichungen sind auf die unterschiedlichen Erhebungsmethoden zurückzuführen. Wie für den HBSC-Studienteil 2005 bis 2006 in Wales bereits festgestellt und publiziert [39], führen subjektive Angaben der Jugendlichen zu Größe und Gewicht zu einer Unterschätzung des BMI und damit automatisch zu einer Unterschätzung des Anteils an Übergewichtigen und Adipösen.

$\mathrm{Zu}$ einem analogen Ergebnis kommen vergleichende Auswertungen der im Rahmen von KiGGS parallel erhobenen subjektiven und objektiven Angaben zu Größe und Gewicht [40, 41]. In KiGGS wurde für eine Unterstichprobe der elf- bis 17-jährigen Teilnehmer vor der Untersuchung Größe und Gewicht abgefragt. Die nachfolgenden Messungen konnten dann dazu dienen, den Grad der Verzerrung zu quantifizieren. Die interessante Erkenntnis, dass die Abweichung des subjektiven $\mathrm{BMI}$ vom gemessenen nicht nur von Alter und Geschlecht der Jugendlichen, sondern insbesondere von ihrem subjektiven Körperselbstbild abhängt, ist die Basis für ein in [41] vorgeschlagenes Korrekturverfahren für die Prävalenzschätzungen. Der Umstand, dass in der HBSC-Studie das 


\begin{tabular}{|c|c|c|c|c|c|c|c|c|}
\hline \multirow{2}{*}{$\begin{array}{l}\text { Referenz- } \\
\text { system }\end{array}$} & \multicolumn{2}{|c|}{ Kromeyer-Hauschild [3] } & \multicolumn{2}{|l|}{ IOTF [11] } & \multicolumn{2}{|l|}{ WHO $[14,15]^{a}$} & \multicolumn{2}{|c|}{ Rolland-Cachera [13] } \\
\hline & Übergewicht ${ }^{b}$ & Adipositas & Übergewicht ${ }^{b}$ & Adipositas & Übergewicht ${ }^{b}$ & Adipositas & Übergewicht ${ }^{b}$ & Adipositas \\
\hline Jungen & $\begin{array}{l}14,9 \%(13,9- \\
16,0)\end{array}$ & $\begin{array}{l}6,1 \% \\
(5,5-6,8)\end{array}$ & $18,4 \%(17,3-19,6)$ & $4,9 \%(4,4-5,4)$ & $23,8 \%(22,7-25,0)$ & $\begin{array}{l}8,6 \%(7,8- \\
9,4)\end{array}$ & $\begin{array}{l}25,1 \%(24,0- \\
26,2)\end{array}$ & $15,7 \%(14,6-16,8)$ \\
\hline Mädchen & $\begin{array}{l}14,7 \%(13,8- \\
15,7)\end{array}$ & $\begin{array}{l}6,1 \% \\
(5,5-6,8)\end{array}$ & $18,2 \%(17,1-19,3)$ & $4,5 \%(4,0-5,1)$ & $20,8 \%(19,7-21,9)$ & $\begin{array}{l}5,9 \%(5,3- \\
6,5)\end{array}$ & $\begin{array}{l}24,9 \%(23,8- \\
26,1)\end{array}$ & $14,6 \%(13,7-15,7)$ \\
\hline
\end{tabular}

subjektive Körperselbstbild ebenso abgefragt wurde wie in KiGGS, eröffnet die Möglichkeit für ein effizientes Korrekturverfahren zur Prävalenzschätzung von Übergewicht und Adipositas [41].

Da davon auszugehen ist, dass der Grad der Unterschätzung des BMI und damit auch das Ausmaß der Fehlklassifikationen für Übergewicht und Adipositas bei den teilnehmenden Ländern der HBSC-Studie unterschiedlich sind, sind auch internationale Vergleiche, wie in [38] dargestellt, nur von eingeschränktem Wert.

\section{Fazit}

Die in Deutschland zur Verfügung stehenden Daten legen nahe, dass die in den letzten Jahren zugenommene Häufigkeit von Adipositas, insbesondere von Adipositas im Kindes- und Jugendalter, ein Ernst zu nehmendes PublicHealth-Problem darstellt. Das hatte eine Vielzahl von gesundheitspolitischen Programmen, Aktivitäten und Maßnahmen mit zum Teil konkreten Zielstellungen zur Folge [42].

Um nun die Zielerreichung beziehungsweise die Fortschritte auf dem Wege zur Verminderung von Übergewicht und Adipositas nachweisen zu können, bietet es sich an, die aktuellsten zur Verfügung stehenden KiGGS-Ergebnisse zur Charakterisierung der Ausgangslage zu verwenden. Schon jetzt werden in Gesundheitsberichten der Kommunen und Länder die Adipositasraten der Schuleingangsuntersuchungen mit den KiGGS-Ergebnissen verglichen. Dies hat den Vorteil, dass man nicht retrospektiv eine Einordnung vornimmt, sondern sich aktuell orientieren kann, ob man sich im Mittelfeld oder im „Spitzenfeld“ der Adipositasraten befindet. Das in [16] publizierte Referenzsystem wird künftig die Ausgangslage definieren und zur Feststellung zeitlicher Trends und zur Bewertung von Ergebnissen dienen. Als Grundlage für die Definition von Übergewicht und Adipositas sollte dagegen weiterhin das Referenzsystem von Kromeyer-Hauschild et al. [3] verwendet werden, um die Prävalenzen nicht künstlich „schön“ zu rechnen. Allerdings sollte das Kromeyer-Hauschild-System für Kinder unter zwei Jahren nicht mehr verwendet werden [16].

Die im Rahmen des Gesundheitsmonitorings zu erwartenden neuen Prävalenzschätzungen (sechs Jahre später) können die Entwicklungen natürlich nur für ganz Deutschland abbilden, und das auch nur in relativ großen Abständen. Daher müssen zusätzlich andere, regional differenzierte Erhebungen, wie beispielsweise die Schuleingangsuntersuchungen, für eine Evaluation herangezogen werden. Diese Erhebungen sind sehr sorgfältig auf ihre Qualität zu prüfen, der Einsatz standardisierter Verfahren sowohl für die Erhebung als auch für die statistische Auswertung muss gewährleistet werden. An der Erreichung dieser methodischen Zielstellungen arbeiten bereits jetzt Arbeitsgruppen der Kinder- und Jugendärzte des ÖGD. Subjektive Angaben der Jugendlichen beziehungsweise ihrer Eltern zur Bestimmung des BMI und damit zur Klassifikation von Übergewicht und Adipositas sind ohne eine zeitgleiche Validierungsstudie nicht zu verwenden. Nach [41] ist zu empfehlen, dass generell Angaben zum Körperselbstbild mit zu erheben sind, um das Ausmaß der Verzerrungen des subjektiven $B M I$ einschätzen und dieses gegebenenfalls korrigieren zu können.

Die vielfältigen Maßnahmen zur Prävention von Adipositas bei Kindern und Jugendlichen in Deutschland wurden un- ter anderem dadurch initiiert, dass die epidemiologische Datenlage alle Warnleuchten „,auf Rot" gehen ließ. Es sollte nunmehr wiederum über qualitätsgesicherte epidemiologische Erhebungen begleitend festgestellt werden, ob diese Maßnahmen über einen längeren Zeitraum gesehen auch wieder Anlass zur Entwarnung geben können. Damit bleibt die Frage nach der Verbreitung von Übergewicht und Adipositas bei Kindern und Jugendlichen auch über die nächsten Dekaden von Interesse.

\section{Korrespondenzadresse}

\section{Dr. B.-M. Kurth}

Robert Koch-Institut

13303 Berlin

kurthb@rki.de

\section{Literatur}

1. Wabitsch M (2004) Kinder und Jugendliche mit Adipositas in Deutschland. Aufruf zum Handeln. Bundesgesundheitsbl Gesundheitsforsch Gesundheitsschutz 47:251-255

2. Kurth BM, Schaffrath Rosario A (2007) Die Verbreitung von Übergewicht und Adipositas bei Kindern und Jugendlichen. Bundesgesundheitsbl Gesundheitsforsch Gesundheitsschutz 50(5/6):736-743

3. Kromeyer-Hauschild K, Wabitsch M, Kunze D et al (2001) Perzentile für den Body-Mass-Index für das Kindes- und Jugendalter unter Heranziehung verschiedener deutscher Stichproben. Monatsschr Kinderheilkd 149:807-818

4. Dietz W, Robinson TN (1998) Use of the body mass index (BMI) as a measure of overweight in children and adolescents. J Pediatr 132:191-193

5. Zwiauer K, Wabitsch M (1997) Relativer BodyMass-Index (BMI) zur Beurteilung von Übergewicht und Adipositas im Kindes- und Jugendalter. Empfehlungen der European Childhood Obesity Group. Monatsschr Kinderheilkd 145:1312-1318

6. Hirschler V, Ruiz A, Romero T et al (2009) Comparison of different anthropometric indices for identifying insulin resistance in schoolchildren. Diabetes Technol Ther 11:615-621

7. Plachta-Danielzik S, Landsberg B, Johannsen M et al (2008) Association of different obesity indices with blood pressure and blood lipids in children and adolescents. Br J Nutr 100:208-218 
8. Freedman DS, Kahn HS, Mei Z et al (2007) Relation of body mass index and waist-to-height ratio to cardiovascular disease risk factors in children and adolescents: the Bogalusa Heart Study. Am J Clin Nutr 86:33-40

9. Kleiser C (2010) Determinants and health risks of overweight and obesity among children and adolescents in Germany. Dissertation, Rheinische Friedrich-Wilhelms-Universität, Bonn. http://hss. ulb.uni-bonn.de/2010/2038/2038.htm

10. Garnett SP, Baur LA, Srinivasan S et al (2007) Body mass index and waist circumference in midchildhood and adverse cardiovascular disease risk clustering in adolescence. Am J Clin Nutr 86:549-555

11. Cole TJ, Bellizzi MC, Flegal KM et al (2000) Establishing a standard definition for child overweight and obesity worldwide: international survey. BM 320:1-6

12. Cole TJ, Green P (1992) Smoothing reference centile curves: the LMS method and penalized likelihood. Stat Med 11:1305-1319

13. Rolland-Cachera MF, Cole TJ, Sempe M et al (1991) Body mass index variations: centiles from birth to 87 years. Eur J Clin Nutr 45:13-21

14. WHO Multicentre Growth Reference Study Group (2006) WHO child growth standards based on length/height, weight and age. Acta Paediatr Suppl 450:76-85. See also http://www.who.int/ childgrowth/standards/en/index.html (Stand: 22.1.2010)

15. Onis M de, Onyango AW, Borghi E et al (2007) Development of a WHO growth reference for schoolaged children and adolescents. Bull World Health Organ 85:660-667. See also http://www.who.int/ growthref/en (Stand: 22.1.2010)

16. Schaffrath Rosario A, Kurth BM, Stolzenberg $\mathrm{H}$ et al (2010) Body mass index percentiles for children and adolescents in Germany based on a nationally representative sample (KiGGS 2003-2006). Eur J Clin Nutr 64:341-349

17. Pigeot I, Buck C, Herrmann D et al (2010) Übergewicht und Adipositas bei Kindern und Jugendlichen: Die weltweite Situation. Bundesgesundheitsbl Gesundheitsforsch Gesundheitsschutz 53

18. Sherry B, Jefferds ME, Grummer-Strawn LM (2007) Accuracy of adolescent self-report of height and weight in assessing overweight status: a literature review. Arch Pediatr Adolesc Med 161(12):11541161

19. Currie C, Samdal O, Boyce W, Smith R (eds) (2001) Health behaviour in school-aged children: a WHO cross-national study (HBSC), research protocol for the 2001/2002 survey. Child and Adolescent Health Research Unit (CAHRU), University of Edinburgh

20. Hurrelmann K, Klocke A, Melzer W, Ravens-Sieberer U (2003) Jugendgesundheitssurvey. Internationale Vergleichsstudie im Auftrag der Weltgesundheitsorganisation WHO. Juventa, Weinheim München

21. Richter M, Hurrelmann K, Klocke A et al (Hrsg) (2008) Gesundheit, Ungleichheit und jugendliche Lebenswelten. Ergebnisse der zweiten internationalen Vergleichsstudie im Auftrag der Weltgesundheitsorganisation WHO. Juventa, Weinheim München

22. Kurth $B M$, Kamtsiuris $P$, Hölling $\mathrm{H}$ et al (2008) The challenge of comprehensively mapping children's health in a nation-wide health survey: design of the German KiGGS-Study. BMC Public Health $8(1): 196$
23. Lange $M$, Kamtsiuris $P$, Lange $C$ et al (2007) Messung soziodemographischer Merkmale im Kinderund Jugendgesundheitssurvey (KiGGS) und ihre Bedeutung am Beispiel der Einschätzung des allgemeinen Gesundheitszustands. Bundesgesundheitsbl Gesundheitsforsch Gesundheitsschutz 50(5/6):578-589

24. Schenk L, Ellert U, Neuhauser H (2007) Kinder und Jugendliche mit Migrationshintergrund in Deutschland. Methodische Aspekte im Kinderund Jugendgesundheitssurvey (KiGGS). Bundesgesundheitsbl Gesundheitsforsch Gesundheitsschutz 50(5/6):590-599

25. Kamtsiuris $P$, Lange $M$, Schaffrath Rosario A (2007) Der Kinder- und Jugendgesundheitssurvey (KiGGS): Stichprobendesign, Response und Nonresponse-Analyse. Bundesgesundheitsbl Gesundheitsforsch Gesundheitsschutz 50:547-556

26. SAS Institute Inc (2008) SAS/STAT 9.2 User's Guide. SAS Institute Inc, Cary, NC

27. Kurth BM, Lange C, Kamtsiuris P, Hölling H (2009) Gesundheitsmonitoring am Robert Koch-Institut. Sachstand und Perspektiven. Bundesgesundheitsbl Gesundheitsforsch Gesundheitsschutz 52(5):557-570

28. Buyken AE, Hahn S, Kroke A (2005) Differences between recumbent length and stature measurement in groups of 2-and 3-y-old children and its relevance for the use of European body mass index references. Int J Obes 29:24-28

29. Kuczmarski RJ, Ogden CL, Grummer-Strawn LM et al (2000) CDC Growth charts: United States. Advance data from vital and health statistics; no. 314 National Center for Health Statistics, Hyattsville, Maryland, US

30. Statistisches Bundesamt: Fortschreibung des Bevölkerungsstandes. http://www.destatis.de, zuletzt zugegriffen am 15.10.2009

31. Robert Koch-Institut (2008) Beiträge zur Gesundheitsberichterstattung: Kinder- und Jugendgesundheitssurvey (KiGGS) 2003-2006: Kinder und Jugendliche mit Migrationshintergrund in Deutschland. Robert Koch-Institut, Berlin

32. Kleiser C, Schaffrath Rosario A, Mensink GB et al (2009) Potential determinants of obesity among children and adolescents in Germany: results from the cross-sectional KiGGS study. BMC Public Health 9:46

33. Moß A, Wabitsch M, Kromeyer-Hauschild K et al (2007) Prävalenz von Übergewicht und Adipositas bei deutschen Einschulkindern. Bundesgesundheitsbl Gesundheitsforsch Gesundheitsschutz 50(11):1424-1431

34. Schaffrath Rosario A, Kurth BM (2009) Regionale Unterschiede in der Häufigkeit von Übergewicht und Adipositas bei deutschen Einschülern. Realität oder Artefakt? Bundesgesundheitsbl Gesundheitsforsch Gesundheitsschutz 52(6):643-646

35. Simon K, Rosenkötter N (2010) Adipositas bei Einschülern in Nordrhein-Westfalen. Tatsächliche Stagnation des Anteils adipöser Einschüler oder Fehlinterpretation? Bundesgesundheitsbl Gesundheitsforsch Gesundheitsschutz 53:258-264

36. Apfelbacher CJ, Cairns J, Bruckner T et al (2008) Prevalence of overweight and obesity in East and West German children in the decade after reunification: population-based series of cross-sectional studies. J Epidemiol Community Health 62(2):125130
37. Hähne C, Dümmler K (2008) Einflüsse von Geschlecht und sozialer Ungleichheit auf die Wahrnehmung und den Umgang mit dem Körper im Jugendalter. In: Richter M, Hurrelmann K, Klocke A et al (Hrsg) Gesundheit, Ungleichheit und jugendliche Lebenswelten. Ergebnisse der zweiten internationalen Vergleichsstudie im Auftrag der Weltgesundheitsorganisation WHO. Juventa, Weinheim München, S 93-115

38. Haug E, Rasmussen M, Samdal O et al (2009) Overweight in school-aged children and its relationship with demographic and lifestyle factors: results from the WHO-Collaborative Health Behaviour in School-aged Children (HBSC) Study. Int J Public Health 54:S167-S179

39. Elgar FJ, Roberts C, Tudor-Smith C, Moore L (2005) Validity of self-reported height and weight and predictors of bias in adolescents. J Adolesc Health 37(5):371-375

40. Kurth BM, Ellert U (2008) Gefühltes oder tatsächliches Übergewicht: Worunter leiden Jugendliche mehr? Ergebnisse des Kinder- und Jugendgesundheitssurveys KiGGS. Dtsch Arztebl 105(23):406412

41. Kurth BM, Ellert U (2010) Estimated and measured $\mathrm{BMI}$ and self perceived body image of adolescents in Germany - general implications for correcting prevalence estimations of overweight and obesity. Obes Facts (in press)

42. Robert Koch-Institut und Bundeszentrale für gesundheitliche Aufklärung (Hrsg) (2008) Erkennen Bewerten - Handeln: Zur Gesundheit von Kindern und Jugendlichen in Deutschland. RKI und BZgA, Berlin 